\title{
Synthetic Vascular Ultrasound Imaging Through Coupled Fluid-Structure Interaction and Ultrasound Simulations
}

\author{
A. Swillens ${ }^{1}$, J. Degroote $^{2}$, J. Vierendeels ${ }^{2}$, L. Lovstakken ${ }^{2}$, and P. Segers ${ }^{1}$ \\ ${ }^{1}$ IBiTech-bioMMeda, Ghent University, Gent, Belgium \\ ${ }^{2}$ Department of flow, heat and combustion mechanics, Ghent University, Gent, Belgium \\ ${ }^{3}$ Department of Circulation and Medical Imaging, Norwegian University of Science and Technology, Trondheim, Norway
}

\begin{abstract}
Although ultrasonic imaging is commonly applied in cardiovascular research and clinical practice, current blood flow and vessel wall imaging methods are still hampered by several limitations. We developed a simulation environment integrating ultrasound (US) and fluid-structure interaction (FSI) simulations, allowing construction of synthetic USimages based on physiologically realistic behavior of an artery. An in-house code was developed to strongly couple the flow solver Fluent and structural solver Abaqus using an Interface Quasi-Newton technique. A distensible tube, representing the common carotid artery (length $5 \mathrm{~cm}$, inner diameter $6 \mathrm{~mm}$, thickness $1 \mathrm{~mm}$ ), was simulated. A mass flow inlet boundary condition, based on flow measured in a healthy subject, was applied. A downstream pressure condition, based on a noninvasively measured pressure waveform, was used. USsimulations were performed with Field II, allowing to model realistic transducers and scan sequences as used in clinical vascular imaging. To this end, scatterers were "seeded" in the fluid and structural domain and propagated during the simulated scan procedure based on flow and structural displacement fields from FSI. Simulations yielded raw ultrasound (RF) data, which were processed for arterial wall distension and shear rate imaging. Our simulations demonstrated that (i) the wall distension application is sensitive to measurement location (highest distension found when tracking the intima-lumen transition); (ii) strong reflections between tissue transitions can potentially cloud a correct measurement; (iii) maximum shear rate was underestimated during the complete cardiac cycle, with largest discrepancy during peak systole; (iv) due to difficulties measuring near-wall velocities with US, shear rate reached its maximal value at a distance from the wall $(0.812$ $\mathrm{mm}$ for anterior and $\mathbf{0 . 6 8 9} \mathrm{mm}$ for posterior side). We conclude that our FSI-US simulation environment provides realistic RF-signals which can be processed into ultrasound-derived medical images and measurements.
\end{abstract}

Keywords - Biomechanics, multi-physics, ultrasound, blood flow, fluid-structure interaction.

\section{INTRODUCTION}

At present, ultrasonic echography is still the preferred method for clinical screening of atherosclerosis in large, superficial arteries. In particular, the carotid artery is often investigated in clinical protocols since the artery directly supplies blood to the brain and the location is prone to development of atherosclerosis. However, most commonly used clinical blood flow imaging modalities are still limited to $1 \mathrm{D}$ measurements since only the velocity component in the direction of the ultrasound beam is visualized. Other imaging modalities aim to assess arterial stiffness, but here the current methods are also hampered. A common application is measuring tissue velocities using Doppler based methods, which is limited to $1 \mathrm{D}$ visualization as well. Often, tissue velocities are further integrated to assess vessel distension. However, vessel kinematics rather than vessel mechanics is hence assessed.

We recently developed a computer simulation tool [1] which integrates computational fluid dynamics (CFD) with ultrasound simulation (US) software (Field II; [2]), providing a flexible tool for the validation and development of ultrasound blood flow modalities in the presence of complex flow. The ultrasonic echoes are simulated by modeling blood as a random point scatterer distribution on which the ultrasound waves reflect. The scatterer positions are updated using the CFD-velocity fields interpolated in space and time. An important limitation of our work, however, was the absence of the moving vessel wall in our model, which influences flow visualization through the motion of the boundaries of the fluid domain, as well as via ultrasound echoes generated by the vessel wall. Fluid-structure interaction (FSI) simulations allow for the coupled computation of blood flow and arterial wall mechanics [3]. It is therefore a natural extension of our work to also seed scatterers in the vessel wall, and to use FSI-simulations to calculate scatterer positions in both the blood flow and vessel wall.

The aim of this paper is two-fold. We first present a method providing scatterer phantoms of both the blood flow and arterial wall, i.e., a virtual echographic phantom of a straight arterial segment. Subsequently, we illustrate the potential of the model. As a first application, the ultrasonic measurement of vessel distension will be investigated [4]. We will use the RF-data resulting from scatterers in the arterial wall to assess the performance of a previously used vessel wall-tracking algorithm. In a second application, we 
will study to what extent it is possible to measure velocity and shear rate profiles using Doppler-based measurements along one scanline. As earlier described by Brands et al. [5], shear rate profiles can be derived from flow velocity profiles measured with ultrasound. However, due to difficulties in measuring the low velocities in the vicinity of the moving wall, the maximal shear rate is measured at a certain distance from the wall, while the actual maximal shear rate might be differing from this position and in magnitude. To the best of our knowledge, the relationship between ground-truth and ultrasound-derived shear rate has never been studied in detail.

\section{MethodS}

\section{A. Fluid-structure interaction simulations}

FSI-simulations were performed in a partitioned way, computing the flow and structural equations with a separate flow and structural solver [3]. An in-house code 'Tango' was used to couple the flow solver Fluent (Ansys, Canonsburg, PA, USA) and the structural solver Abaqus (Simulia,Inc., Providence, RI, USA). In particular, DirichletNeumann partitioning was used (flow problem is solved for a given displacement; structural problem is solved for a stress boundary condition applied on wet side of the structure). To enhance convergence of the coupling iterations, an Interface Quasi-Newton method was used, which replaces the complex fluid or solid solver on the interface by approaching the Jacobian of the solver on the interface [3]. Further, an Arbitrary Lagrangian Eulerian (ALE) method was used to match the different grid formulations for the fluid and structural domain.

A straight elastic tube with inner radius $3 \mathrm{~mm}$, outer radius $4 \mathrm{~mm}$, and length $5 \mathrm{~cm}$ was simulated. The vessel wall was modeled using a linearized elastic material model with a Young modulus of $250 \mathrm{kPa}$, Poisson modulus of 0.49 , and density $1200 \mathrm{~kg} / \mathrm{m}^{3}$. Linearization was performed with respect to a reference pressure and inner radius of respectively $10 \mathrm{mmHg}$ and $3 \mathrm{~mm}$. Circumferential and longitudinal movement of the tube was prevented. Blood was modeled as a Newtonian liquid with a viscosity of $3.5 \mathrm{mPas}$ and a density of $1050 \mathrm{~kg} / \mathrm{m}^{3}$. A velocity profile was measured in the common carotid artery of a healthy volunteer using ultrasonic pulsed wave Doppler (12L linear array vascular probe, GE Medical Systems, Milwaukee, WI, USA) and was applied as a mass flow inlet condition. The outlet boundary condition was a non-invasively measured pressure waveform, scaled with a pulse pressure of $40 \mathrm{mmHg}$, representative of a healthy hemodynamical condition.

The fluid mesh consisted of 34400 triangular prisms and was adapted to the position of the fluid-structure interface
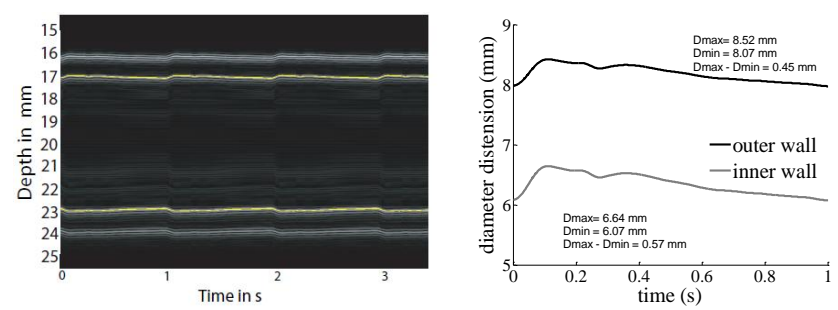

Fig. 1 Left: M-mode display of RF-data designating arterial wall motion. Right: diameter distension waveforms after tracking the motion of the lumenintima (inner wall) and media-adventitia (outer wall) transition.

with a spring analogy. The structural domain was composed of 720 quadratic continuum hexahedrons (20 nodes) and numerical damping of high-frequency errors was increased $(\alpha=-0.3)$ to eliminate spurious oscillations due to the different time discretizations in Fluent and Abaqus. Hexahedral elements were used to allow layered modeling of the vessel wall. A non-matching mesh existed on the interface requiring appropriate interpolation of the transfer variables. The cardiac cycle of $1 \mathrm{~s}$ was divided into timesteps of $5 \mathrm{~ms}$ and 2 cycles were computed to obtain results independent of transient effects.

\section{B. Simulating ultrasound using Field-II}

The RF-signals from the vessel wall and blood were simulated using the Field II software created by Jensen et al. [2]. This simulation software allows modeling arbitrary ultrasound transducers and realistic image scan sequencing. Using linear system theory, the ultrasound field is determined based on the ultrasonic excitation pulse, the temporal impulse responses of the transmitting and receiving transducers, and the spatial impulse response at a given point. Field II models tissue as a collection of random point scatterers. The required scatterer density is related to the imaging system resolution, with 10 scatterers per resolution cell assuring Gaussian distributed RF-signals. The scattering strength is modeled using a normal distribution of scattering amplitudes with mean and standard deviation varying according to the tissue properties. To appropriately mimic specular reflections, scatterers with high mean amplitude are positioned in a regular fashion at the tissue transitions. Dynamic objects are achieved by moving the point scatterers during simulation. Each ultrasound beam is simulated individually, and it is therefore possible to update the position of moving scatterers between beam acquisitions. 


\section{Constructing the scatterer phantom}

We previously developed a method to generate scatterers for Field II simulations based on CFD-calculations with rigid walls [1]. For distensible fluid geometries, however, straightforward linear temporal interpolation of the velocity fields is however not possible. We therefore followed an approach where the scatterer displacement is approximated by updating scatterer velocities for each FSI-timestep. To avoid that scatterers are displaced outside the fluid domain in a shrinking geometry or that voids are created in an expanding geometry, scatterers are displaced using the velocity vector from the subsequent FSI time step, with the velocity vector extracted from an approximated mapped position at that time step. This approach is justified due to the Backward Euler time discretization used by the flow solver. It provides correct displacements for scatterers at the fluid-structure interface, but it is an approximated approach within the flow field.

The structure phantom generation is less complex because of the Lagrangian grid formulation. The grid displacement corresponds with the material displacement and hence also with the scatterer displacement. However, the vessel wall needs more refined scatterer generation due to its complex composition, with flexibility of defining different scattering properties in different vessel regions. Therefore, the mesh was divided into 3 layers of hexahedrons, with scatterer properties easily modifiable for each layer. This represents to some extent the intima, media and adventitia of an artery, although we assumed equal thickness of each layer, while this is not the case in vivo. Besides these
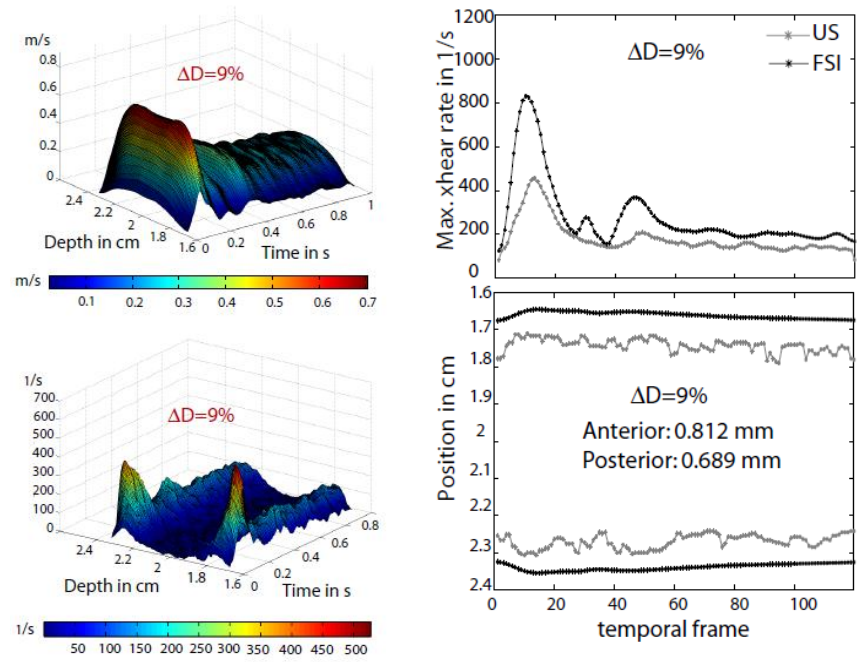

Fig. 2 Left: angle-corrected velocities derived from the RF-data using an autocorrelation velocity estimator (top) and derived shear rate profile (bottom). Right: comparison between peak shear rate directly derived from the

FSI-data and obtained from the ultrasound data (top) and display of the position where maximum shear rate is found with FSI and US. random scatterers, we also mimicked the specular reflections at the transition regions between different tissue types (i.e. tissue/vessel wall and vessel wall/blood) by placing scatterers at fixed distances along these interfaces. These mimicked specular reflections had a higher intensity than the scattering from the random tissue and blood scatterers.

\section{Ultrasound applications}

The final result of the simulation is a dataset consisting of raw ultrasound (RF-data) signals, generated from the interaction of an emitted ultrasound field and the moving scatterers in the fluid and structural domain. We illustrate the potential of the model via two vascular imaging applications.

(i) In a first application, we simulate the ultrasonic measurement of vessel distension, where the ultrasound beam is transmitted perpendicular to the vessel wall. Vessel wall motion is tracked by integrating wall velocities: $\mathrm{z}[\mathrm{t}+\Delta \mathrm{t}]=\mathrm{z}[\mathrm{t}]+\mathrm{v}[\mathrm{t}] \Delta \mathrm{t}$, with $\mathrm{z}[\mathrm{t}]$ the position in the vessel wall, $\mathrm{v}[\mathrm{t}]$ as estimated by ultrasound (modified autocorrelation approach), and $\Delta \mathrm{t}$ the velocity resolution corresponding to the packet size times the pulse repetition period $(3 \cdot 1 / 1000)$. Only the RF-data of the arterial wall are processed for this application, hence neglecting a potential effect of the flow motion on the wall velocity estimator. A 12L linear array probe (GE Medical Systems, Milwaukee, WI, USA), as used in the applied distension software [4], was modeled with a 1.5 period sinusoidal pulse excitation of $8 \mathrm{MHz}$ centre frequency. Due to the lower velocity range of tissue compared to blood, a lower PRF of $1 \mathrm{kHz}$ was applied.

(ii) For the flow (shear rate) application, the tube phantom was angled 70 degrees with respect to the ultrasound beam (=axial) direction. Velocity profiles in the axial direction were obtained using an autocorrelation algorithm. Note that only the RF-signal from the blood was used to estimate flow velocities. A 4-period sinusoidal excitation pulse with $5 \mathrm{MHz}$ centre frequency was chosen. Velocity profiles halfway the tube were obtained using a packet of 64 pulses emitted with a PRF of $8 \mathrm{kHz}$, resulting in 120 frames for the complete cardiac cycle. A sliding window averaging filter was used. In the results, displayed velocities are anglecorrected velocities and thus represent velocities along the axis of the tube.

\section{RESULTS}

\section{A. Measurement of vessel distension}

RF ultrasound data for the wall were generated from the FSI-US coupling procedure, and are displayed as an $\mathbf{M -}$ 
Mode image in Fig. 1. Using the distension software of Rabben et al. [4], the motion of the anterior and posterior intima-lumen ("inner wall" tracking, see Fig 1.) and mediaadventitia ("outer wall" tracking) was tracked. Subtracting these tracking curves yielded the vessel diameter distension waveforms, as displayed in Fig. 1. Using inner wall tracking data, arterial distension $\Delta \mathrm{D} / \mathrm{D} \quad(\Delta \mathrm{D}=$ Dmax-Dmin and $\mathrm{D}=\mathrm{Dmin}$ ) was $9 \%$, which is representative for the distension of the common carotid artery of a healthy adult. There was an excellent match between diameter distension data obtained from the FSI-computations (reference) and the ultrasound wall tracking data for the inner and outer wall tracking, while scatter was introduced when the tracking was based on points within the vessel wall.

\section{B. Measurement of wall shear rate}

Fig. 2 shows the two-dimensional velocity (anglecorrected values) and derived shear rate distribution in depth and time, obtained from the FSI-US coupling procedure. FSI-US shear rate reaches its maximum value at a certain distance from the wall. FSI-US derived maximal shear rate is systematically lower than the reference value derived from the FSI simulations, with the largest discrepancy appearing near peak systole. There was no clear relation between the location of maximal shear rate and the actual position of the arterial wall. However, one can notice that the position of the ultrasound-derived maximal shear rate shows an asymmetrical trend compared to the vessel centre: maximal shear rate is measured closer to the posterior wall than to the anterior wall.

\section{DISCUSSION AND CONCLUSIONS}

Although the multiphysics model as presented in this study represents a major improvement over our existing model, limitations are still present, both at the biomechanical and acoustical level. More realistic material behavior including anisotropic and hyperelastic material properties could have been modeled (at the expense of higher computational times). The influence of the surrounding tissue on the vessel wall movement, residual stresses and effects of longitudinal pre-stretch of the structure could be taken into account in future work. Further, the boundary conditions to solve the fluid problem could be refined by applying a physiologically realistic vascular impedance as outlet boundary condition, inducing more realistic wave propagation phe- nomena in the tube. The Field II method does not account for nonlinear wave propagation of ultrasound waves or multiple scattering. We did not include noise or frequency dependent scattering in our simulations. Furthermore, the tissue echogenic properties are simulated as a combination of random scatterers in the wall and mimicked specular reflections along the inner and outer boundary of the wall. Although this results in realistically looking RF spectra, further fine-tuning and optimization towards RF-spectra of actual tissue may be mandatory. It should also be stressed that the distension data are solely based on RF-signals from the wall, while the shear rate application relied only on RFsignals from the flow. The shown applications should therefore mainly be considered as a demonstration of the potential of the simulation tool in developing vascular imaging tools, rather than a thorough validation of each of these applications. It is, for that matter, possible that the imaging and signal processing setups can be further optimized for each specific application.

We conclude that our method to couple fluid-structure interaction and ultrasound simulations provides realistic radio-frequent signals from both the tissue and the blood pool which can be processed into ultrasound-derived medical images and measurements. Further research will focus on applications for the ultrasonic investigation of the carotid bifurcation.

\section{REFERENCES}

1. Swillens A, Lovstakken L, Kips J, Torp H and Segers P. (2009). Ultrasound Simulation of Complex Flow Velocity Fields Based on Computational Fluid Dynamics. IEEE TUFFC 56, 546-556.

2. Jensen JA (1996). Field: A program for simulating ultrasound systems. Med. Biol. Eng. Comput. 34, 351-353

3. Degroote J, Bathe KJ and Vierendeels J (2009). Performance of a new partitioned procedure versus a monolithic procedure in fluid-structure interaction. Computers \& Structures 87, 793-801

4. Rabben SI, Bjaerum S, Sorhus V and Torp H. (2002) Ultrasound based vessel wall tracking: an autocorrelation technique with RF center frequency estimation. Ultrasound in Med. \& Biol. 28, 507-517.

5. Brands PJ, Hoeks APG, Hofstra L and Reneman RS (1994). A noninvasive method to estimate wall shear rate using ultrasound. Ultrasound in Med. \& Biol. 21, 171-185

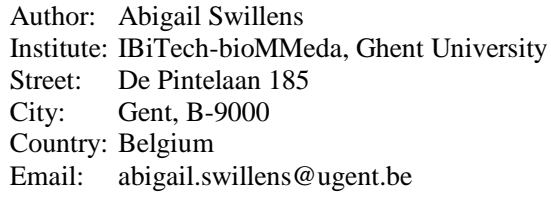

